\title{
Rare case of Richter's syndrome localization in liver and thyroid of a patient with a chronic lymphocytic leukemia (CLL) - Case report and literature
}

\author{
Ewa Wąsik-Szczepanek ${ }^{1, A-F \oplus}$, Agnieszka Szymczyk ${ }^{2, A-F} \oplus$, Dariusz Szczepanek ${ }^{3, A-F}$, \\ Ewelina Grywalska ${ }^{4, C-F \oplus}$, Justyna Szumiło ${ }^{5, B-C, E-F}{ }^{\infty}$, Piotr Trojanowski ${ }^{6, E-F} \oplus$, Olga Czabak ${ }^{1, D-F} \oplus$, \\ Marek Hus ${ }^{1, A, E-F} \oplus$ \\ ${ }^{1}$ Chair and Department of Haematooncology and Bone Marrow Transplantation, Medical University, Lublin, Poland \\ ${ }^{2}$ Department of Clinical Transplantology, Medical University, Lublin, Poland \\ ${ }^{3}$ Chair and Department of Neurosurgery and Paediatric Neurosurgery, Medical University, Lublin, Poland \\ ${ }^{4}$ Chair and Department of Clinical Immunology, Medical University, Lublin, Poland \\ ${ }^{5}$ Chair and Department of Clinical Pathomorphology, Medical University, Lublin, Poland \\ ${ }^{6}$ Department of Otolaryngology and Laryngological Oncology, Medical University, Lublin, Poland \\ A - Research concept and design, B - Collection and/or assembly of data, C - Data analysis and interpretation, \\ $D$ - Writing the article, $E$ - Critical revision of the article, F- Final approval of article
}

Wąsik-Szczepanek E, Szymczyk A, Szczepanek D, Grywalska E, Szumiło J, Trojanowski P, Czabak O, Hus M. Rare case of Richter's syndrome localization in liver and thyroid of a patient with a chronic lymphocytic leukemia (CLL) - Case report and literature. Ann Agric Environ Med. 2020; 27(1): 160-164. doi: 10.26444/aaem/106041

\section{Abstract}

Richter's syndrome (RS) is a rare complication in which chronic lymphocytic leukaemia (CLL) or small lymphocytic lymphoma (SLL) transforms into a more aggressive type of lymphoma - diffuse large B cell lymphoma (DLBCL), or Hodgkin's lymphoma $(\mathrm{HL})$. The review describes the clinical case of a patient with CLL and RS diagnosis. A computed tomography (CT) scan of the abdominal cavity detected numerous normodense areas in the liver. Simultaneously, ultrasound examination (USG) of the thyroid revealed the presence of a solid hypoechogenic lump. The material sampled from closed biopsies of liver and thyroid in both cases allowed the diagnosis of diffuse large B cell lymphoma (DLBCL). The liver and the thyroid are particularly rare locations of RS. However, those cases allowed the conclusion that RS may occur even in a very unexpected and less probable location.

\section{Key words}

liver, thyroid, chronic lymphocytic leukemia, Richter's syndrome

\section{INTRODUCTION}

Chronic lymphocytic leukemia (CLL) is usually a lymphoproliferative disease with a chronic course $[1,2,3]$. It is the most commonly diagnosed type of leukemia in adults. The disease is characterized by the variety of clinical course, prognosis and response to cytostatic treatment; the start of treatment is individual for each patient and depends on the severity of the clinical or progressive nature of the disease. In some patients, mild course and long survival are observed, very often without cytostatic treatment. In the remaining patients, the disease is aggressive and despite intense therapy, it leads to death within 2-3 years.

Richter's syndrome is defined by the transformation of chronic proliferation of B-cells, which is chronic lymphocytic leukemia or lymphocytic lymphoma, into a more aggressive form of lymphoma. Most commonly, it is a diffuse large B-cell lymphoma (DLBCL-RS) [4], rarely Hodgkin lymphoma (Hodgkin variant of RS, HvRS) [5]. The frequency of

Address for correspondence: Agnieszka Szymczyk, Chair and Department of Haematooncology and Bone Marrow Transplantation, Medical University of Lublin, Department of Clinical Transplatology, Medical University of Lublin E-mail: agnieszka.szymczyk.med@wp.pl

Received: 26.06.2018; accepted: 20.02.2019; first published:09.04.2019 occurring of RS among patients with CLL is estimated to be $2 \%-10 \%$ [6] The most frequent RS (80\%-90\%) stems from the same clone as CLL (clonally related RS). In a very small part, the tumor clone develops independently (clonally unrelated RS) [7]. The increased risk of RS occurring is related with the presence of genetic aberrations, such as del 11q and 17p $[8,9]$, unmutated IGHV, significant expression of ZAP-70, CD38 and CD 49d $[10,11]$. RS is characterized by very rapid clinical process. Among more than half of patients (about 59\%) there is fever, weight loss or intensified, night hyperhidrosis [12, 13]. RS most commonly occurs in lymph nodes and bone marrow. Localization in the lymphatic system occurs in about $41 \%$ of cases. Particularly rare cases are those, where transformations occur in the liver and thyroid $[14,3]$.

\section{CASE REPORT}

In June 2007, 60-year-old woman, on the basis of histopathological examination of lymph node and immunophenotype of peripheral blood lymphocytes (CD19+/CD5+/CD23+; Ig lambda+), was diagnosed with chronic lymphocytic leukemia (stage 4 according to Rai classification). Complete peripheral blood count revealed: 
WBC 24.8 K/uL; Lymph. 21.0 K/uL; Hgb 9.9 g/dL; PLT 70.0 K/ul, concentration LDH $132.0 \mathrm{U} / 1$ (10-480). The patient had not reported any general symptoms. Leukemia cells indicated immunological phenotype ZAP70-/CD38+, without the presence of negative genetic aberrations, such as del 11q and del 17p. Physical examination revealed peripheral lymphadenopathy, and USG examination revealed increased size of lymph nodes in the abdominal cavity, without signs of organomegaly. Treatment was started (totally, 6 cycles with chlorambucil and prednisone), resulting in complete remission (CR) of the underlying disease. Afterwards, the patient was observed on an outpatient basis.

In February 2015, the patient was admitted to a hospital due to gradually increasing upper abdominal pain, anorexia and weight loss which had lasted for several weeks. Complete peripheral blood count revealed: WBC 9.79 K/uL, Lymph. 6.75 K/uL, Hgb 11.2 g/dL, PLT 133.0 K/ $\mathrm{uL}$ and $\mathrm{LDH}$ 753.0 U/1. CT examination revealed also an increased liver size (200 $\mathrm{mm}$ in midclavicular line), with irregular outline, displacing neighbouring organs. Reduced vascular pattern, organ parenchyma with density about 40 u.H., almost the whole covered by numerous, segmentally confluent, round normodense areas, showing minor contrast enhancement. Areas of a similar character had been also observed on an increased in size (177mm) spleen (Fig. 1). Furthermore, there were observed numerous, increased in size, lymph nodes located in retroperitoneum. Leukemia lymphocytes indicated phenotype ZAP70-/CD38+ with increased, compared to the time of CLL diagnosis, share of CD38+ cells (36\% vs $49 \%$ ), again without presence of genetic aberrations. Biochemical examinations also revealed increased activity of transaminases: Alat 104.0 U/1 (5-45) and Aspat 161.0 U/1 (5-50). Patient also had closed core biopsy of the liver and a sample of the material was taken for histopathological examination. The biopsy revealed a large lymphoid cells infiltration with morphology of centroblasts and immunoblasts (CD20+; bcl6+; CD10-; bcl2-; CD3-; CD5-; cyclin D1-; Ig lambda+; Ki67 about 90\%). Results of immunohistochemical examinations led to diagnose the transformation of CLL into diffuse large B-cell lymphoma - Richter's syndrome (Fig. 2).

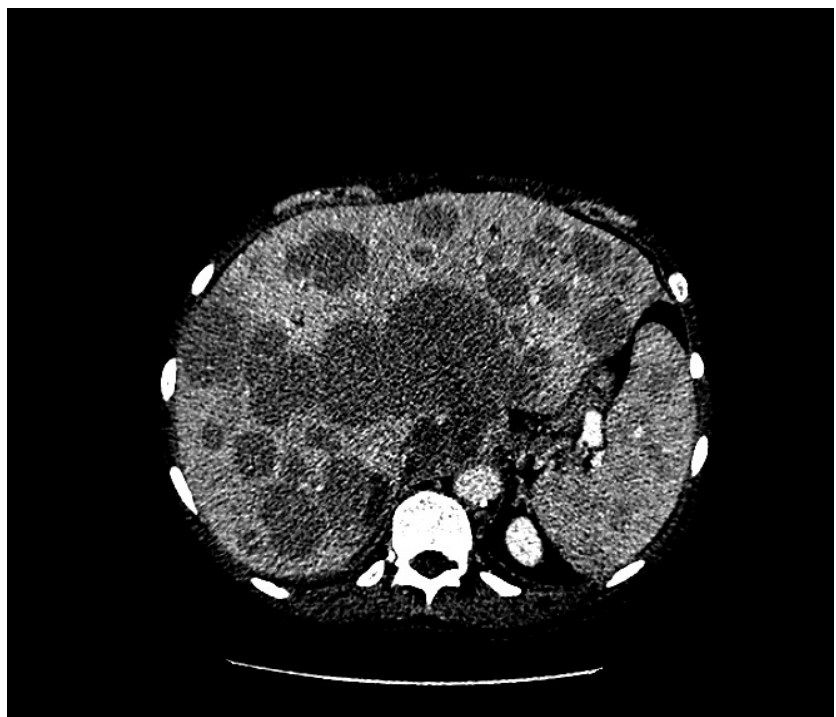

Figure 1. CT scan of the abdomen. Significantly enlarged liver with irregular contours, displacing neighbouring organs. Normodense circular areas in the liver and spleen
At the same time, CT of the chest suggested increased size of thyroid with presence of subcarinal set of lymph nodes (sized $40 \times 30 \mathrm{~mm}$ ). USG examinations of the thyroid revealed the presence of a hypoechogenic lump in the left thyroid lobe and isthmus of the thyroid gland. Concentration of hormones was equal to: TSH $1.67 \mathrm{uIU} / \mathrm{ml}(0.27-4.2) ; \mathrm{FT} 40.95 \mathrm{ng} / \mathrm{dl}$ (9.0-20.0); FT3 $2.57 \mathrm{pg} / \mathrm{ml}$ (4.0-8.3). In the course of the performed fine-needle biopsy, a sample of thyroid and nearby lymph nodes were taken for histopathological examination. Immunohistochemical reactions of the large lymphoid cells infiltration presented the following expression: CD20+, bcl6+, CD10-, bcl2-, CD5-, CD3-, Ki67 about 90\%. The final diagnosis indicated diffuse large B-cell lymphoma in both samples (Fig. 3).

From March - November 2015, the patient received 8 cycles of R-CHOP immunochemotherapy. In December, after performing evaluation of clinical condition of the patient (CT scan, punch biopsy), complete remission was observed of the underlying disease. The patient is still under clinical observation.

\section{DISCUSSION}

Richter's syndrome is defined by transformation of chronic proliferation of B-cells, which is chronic lymphocytic leukemia or lymphocytic lymphoma, into a more aggressive form of lymphoma. Most commonly, it is a diffuse large B-cell lymphoma (DLBCL-RS) [4], rarely Hodgkin lymphoma (Hodgkin variant of RS, HvRS) [5]. The frequency of occurrence of RS among patients with CLL is estimated to be $2 \%-10 \%$ [6] The most frequent RS (80\%-90\%) stems from the same clone as CLL (clonally related RS). In a very small part, a tumour clone develops independently (clonally unrelated RS) [7]. The increased risk of RS occurring is related with presence of genetic aberrations, such as del $11 \mathrm{q}$ and $17 \mathrm{p}[8,9]$, unmutated IGHV, significant expression of ZAP70, CD38 and CD49d $[10,11]$. RS is characterized by very rapid clinical process. Among more than a half of patients (about 59\%) there is fever, weight loss or intensified, night hyperhidrosis $[12,13]$. RS most commonly occurs in lymph nodes and bone marrow. Localization in lymphatic system occurs in about $41 \%$ of cases [14].

The disease described is a case of rare localization of transformation into RS in the course of CLL disease. Usually among CLL patients, the thyroid is secondarily infiltrated by leukemia cells [15]. Lymphomas constitute only about $2 \%$ of all thyroid tumors, while those with the primary localization in this organ constitute only about $2.5 \%[16,17]$. Normal thyroid gland does not have lymphoid tissue. Its presence signifies ongoing pathological process, usually autoimmune inflammation. The literature presents very narrow number of patients with SLL/CLL and Hashimoto's inflammation or other tumors. We have not found a case of patient with Richter's transformation, either. Hashimoto's inflammation is often associated with occurrence of MALT type lymphoma (mucosa-associated lymphoid tissue; about $23 \%$ of lymphomas appearing on the thyroid) and DLBCL (most commonly occurring primary lymphoma of the thyroid, about $50 \%$ of cases) [18]. Bocian et al. and Reid-Nicholson et al. have stated simultaneous occurrence of CLL/SLL with such thyroid tumors as: medullary and papillary carcinomaand mixed papillarycarcinoma $[19,20]$. Shin et al. and Trumper et al. 


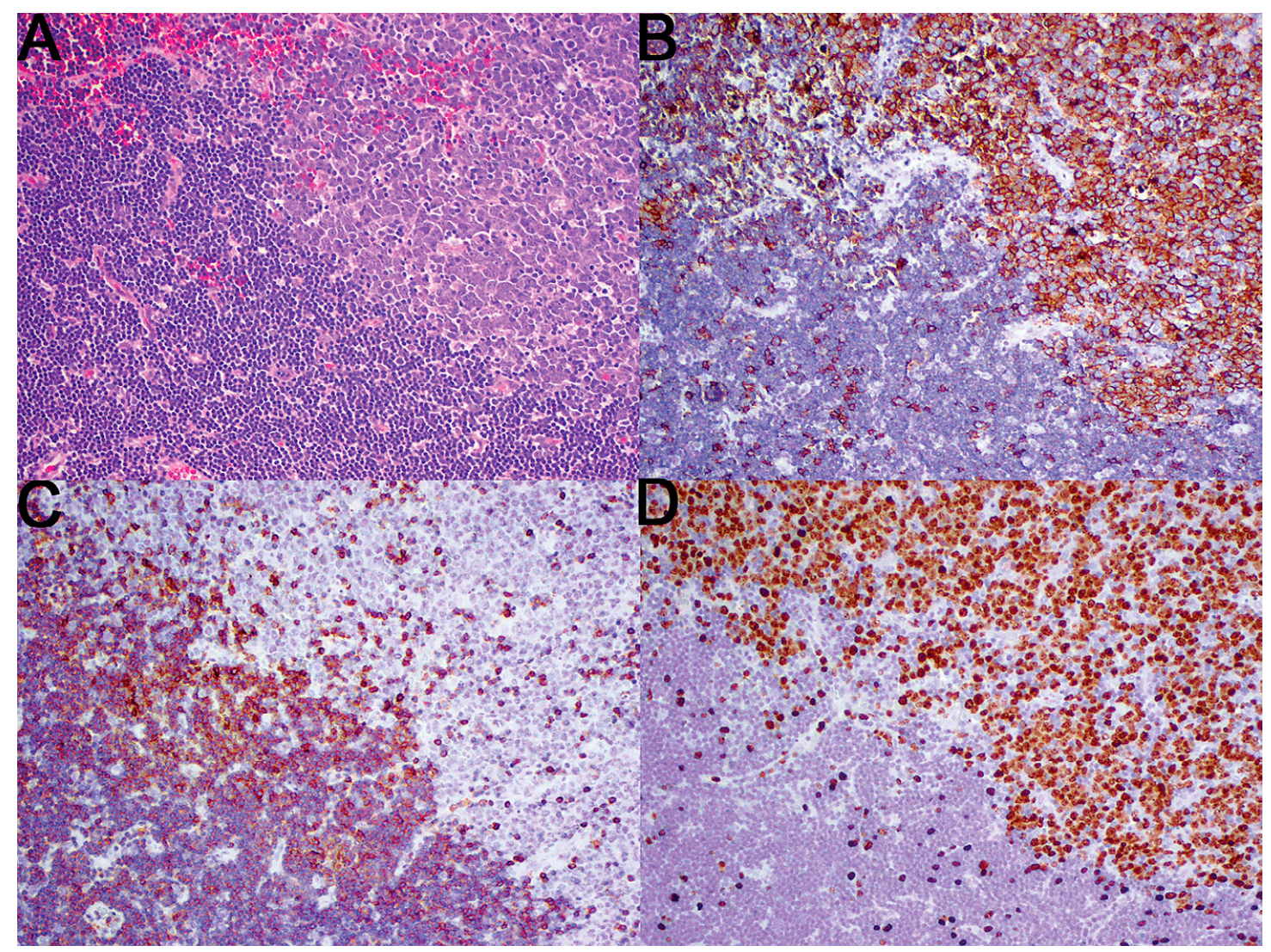

Figure 2. Transformation of chronic lymphocytic leukemia in diffuse large B-cell lymphoma (Richter syndrome) in the liver (A) and immunohistochemical reactions for CD20 (B), CD5 (C) and Ki67 (D) (A - HE; B, C, D - Dako EnVisionTM+/HRP; objective magn. 10x)

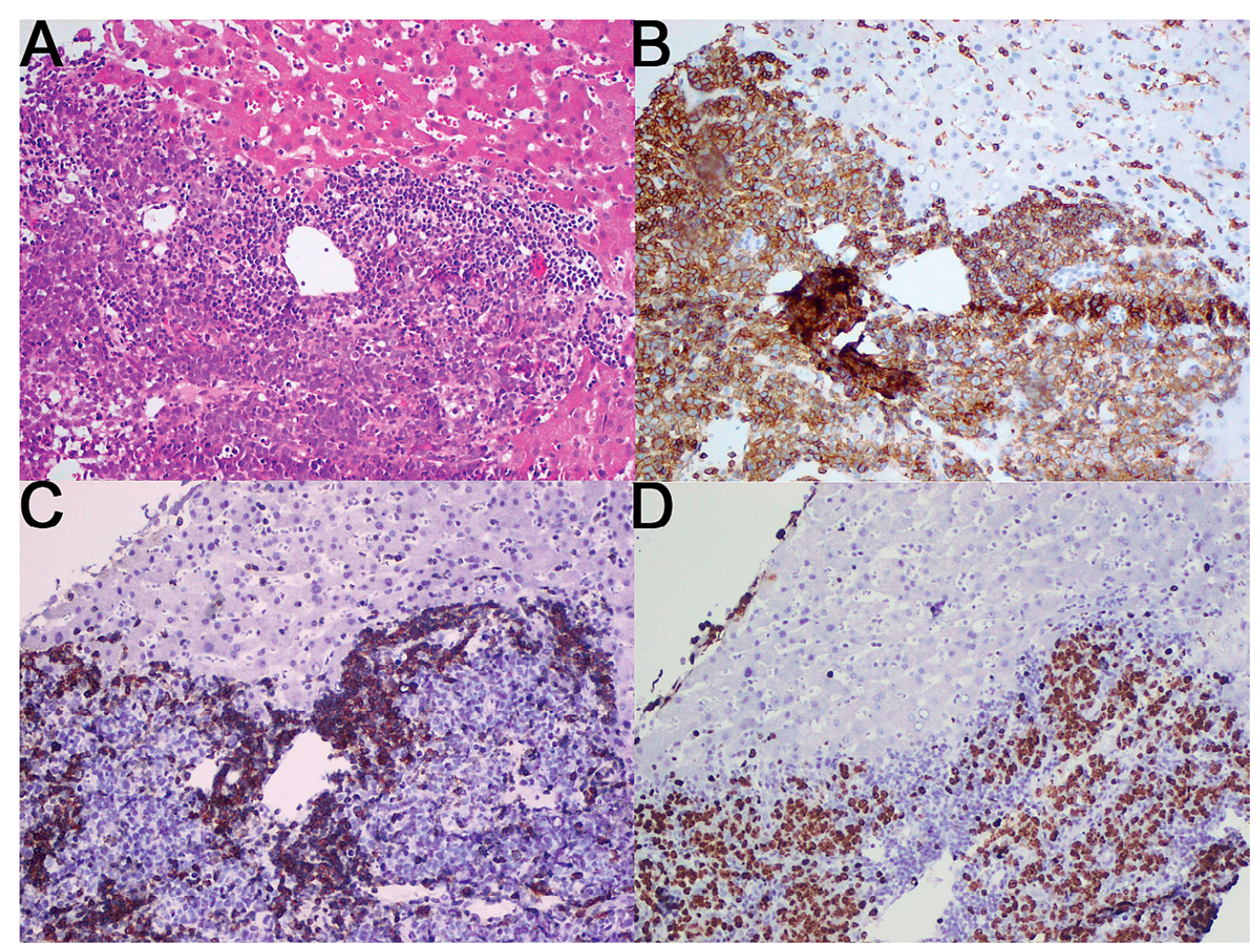

Figure 3. Transformation of chronic lymphocytic leukemia in diffuse large B-cell lymphoma (Richter syndrome) in the lymph node (A) and immunohistochemical reactions for CD20 (B), CD5 (C) and Ki67 (D) (A - HE; B, C, D - Dako EnVisionTM+/HRP; objective magn. 10x) 
presented patients among whom diagnosis of lymphoma had been preceded by autoimmune inflammation with the presence of high concentration of antibodies against peroxidase and thyroglobulin $[21,22]$.

In the patient described in this case report, no preceding or accompanying inflammatory process was confirmed. Decrease in the concentration of FT3 and FT4 could be a result of so-called euthyroid sick syndrome. This phenomenon observed during numerous illnesses, including those related to tumours, however, is not accompanied by dysfunction of the organ itself [23].

In the available literature, only one case was found of RS localization (in the form of diffuse large B cell lymphoma) in liver, described by Tadmor et al. The case concerned a 72-year-old woman, initially treated with chlorambucil and prednisone. Analogously, as in the current case report, the authors observed a higher concentration of transaminases and a significant increase in LDH activity. There was also a very good reaction to immunochemistry therapy, which included vincristine, cyclophosphamide, doxorubicin, prednisolone and rituximab (R-CHOP) [24]. Maryniak et al. presented a case where transformation into Hodgkin lymphoma in the course of CLL was confirmed only after the results of autopsy examination of liver, spleen, adrenal glands and bone marrow [25]. Reddy et al., on the other hand, described HvRS in the liver of 59-year-old woman with a 10-year history of CLL, after many lines of cytostatic treatment (bendamustine, rituximab in monotherapy and with fludarabine). The first, quite unusual, clinical symptom of transformation was a significant increase of bilirubin and alkaline phosphatase concentration [26].

Of specific interest is a case presented by Salihoglu et al. which concerned a 52-year- old woman, where after observation and several more days of chemotherapy (chlorambucil, FC, R-COP), further progression of the disease was confirmed. Because of the detected deletion $17 \mathrm{p}$, the patient was classified for allo-SCT, preceded by 6 CFAR chemotherapy courses (cyclophosphamide, fludarabine, alemtuzumab and rituximab). Clinical and immunophenotypic evaluation of peripheral blood and bone marrow before transplantation indicated complete remission. Repeated FISH test on the day 30 after allo-SCT did not indicate $17 \mathrm{p}$ deletion in bone marrow cells. However, 3 months after transplantation, sudden deterioration occurred in the patient's general health condition: diarrhea, skin lesions, ascites and significant increase of the liver size with the presence of numerous interstitial, focal lesions were all observed.

On the basis of histopathological examination of sample taken during biopsy, large B cell lymphoma was diagnosed, which suggested transformation into RS. The patient died several weeks after confirming RS. According to the authors, the significant factor in transformation into RS, despite accompanying GVHD, was an unfavorable genetic profile of the patient [27].

Attention is being focused on a number of chemotherapy lines. Much controversy has arisen from an opinion about the possible influence of previous treatment for RS development, in particular, specific cytostatic drugs. CLL/SLL ill patients, during their illness usually receive many different types of chemotherapies, therefore the precise definition of a drug with specific significance in this matter is difficult. Modern drugs, including monoclonal antibodies (rituximab, alemtuzumab), may also probably be a significant factor in this process $[28,29]$.

However, in the presented case report, as well as in the case described by Salihoglu et al., the patients are the proof that RS may occur at any moment in the course of the disease, even only after one type of chemotherapy. At the same time, this and other cases indicating the possibility of occurrence of a diffuse large B cell lymphoma among patients with CLL after allo-SCT, always require differentiation with possible post-transplantation lymphoproliferative disorder (PTLPD) $[30,31]$.

\section{CONCLUSIONS}

Richter's syndrome is a rare complication of chronic lymphocytic leukemia, usually with an unfavourable prognosis. The liver and the thyroid are particularly rare locations of RS. On the basis of this case report and studies presented by other authors, it is not possible to clearly determine the influence of the site of transformation on the further course of disease, nor the factors influencing its development. However, such cases allow the conclusion that RS may occur even in the most unexpected and less probable place.

\section{REFERENCES}

1. Rossi D, Spina V, Deambrogi C, et al. The genetics of Richter syndrome reveals disease heterogeneity and predicts survival after transformation. Blood 2011; 117: 3391-3401.

2. Parikh SA, Kay NE, Shanafelt TD. How we treat Richter syndrome. Blood 2014: 123; 1647-1657.

3. Robertson LE, Pugh W, O'Brien S, et al. Richter's syndrome: a report on 39 patients. J Oncology 1993; 11: 1985-1989.

4. Rossi D, Spina V, Deambrogi C, et al. The genetics of Richter syndrome reveals disease heterogeneity and predicts survival after transformation. Blood 2011; 117: 3391-340.

5. Tsimberidou AM, O'Brien S, Kantarjian HM, et al. Hodgkin transformation of chronic lymphocytic leukemia: the M. D. Anderson Cancer Center experience. Cancer 2006; 107: 1294-302.

6. Parikh SA, Kay NE, Shanafelt TD. How we treat Richter syndrome. Blood 2014; 123: 1647-1657.

7. Mao Z, Quintanilla-Martinez L, Raffeld M, et al. IgVH mutational status and clonality analysis of Richter's transformation: diffuse large $\mathrm{B}$-cell lymphoma and Hodgkin lymphoma in association with B-cell chronic lymphocytic leukemia (B-CLL) represent 2 different pathways of disease evolution. Am J Surg Pathol. 2007; 31: 1605-1614.

8. Rossi D, Cerri M, Capello D, et al. Biological and clinical risk factors of chronic lymphocytic leukaemia transformation to Richter syndrome. Br J Haematol. 2008; 142: 202-215.

9. Parikh SA, Rabe KG, Call TG, et al. Diffuse large B-cell lymphoma (Richter syndrome) in patients with chronic lymphocytic leukaemia (CLL): a cohort study of newly diagnosed patients. Br J Haematol. 2013; 162: 774-782.

10. Parikh SA, Shanafelt TD. Risk factors for Richter syndrome in chronic lymphocytic leukemia. Curr Hematol Malig Rep. 2014; 9: 294-299.

11. Jain N. New Developments in Richter Syndrome. Clinical Advances in Hematology \& Oncology 2015; 13: 220-222.

12. Swords R, Bruzzi J, Giles, F. Recent advances in the diagnosis and therapy of Richter's syndrome. Medical Oncology 2007; 24: 17-32.

13. Tsimberidou AM, Keating MJ. Richter syndrome. Biology, incidence, and therapeutic strategies. Cancer 2005; 103: 216-228.

14. Robertson LE, Pugh W, O’Brien S, et al. Richter's syndrome: a report on 39 patients. J Oncol. 1993; 11: 1985-1989.

15. Kossev P, Livolsi V. Lymphoid lesions of the thyroid: review in light of the revised European-American lymphoma classification and upcoming World Health Organization classification. Thyroid 1999; 9: $1273-1280$ 
16. Graff-Baker A, Roman SA, Thomas DC, et al. Prognosis of primary thyroid lymphoma: demographics, clinical, and pathologic predictors of survival in 1,408 cases. Surgery 2009; 146: 1105-1115.

17. Sangalli G, Serio G, Zampatti C, Lomuscio G, Colombo L. Fine needle aspiration cytology of primary lymphoma of the thyroid: a report of 17 cases. Cytopathology 2001; 12: 257-263.

18. Thieblemont C, Mayer A, Dumontet C, et al. Primary thyroid lymphoma is a heterogeneous disease. J Clin Endocrinol Metab. 2002; 87: 105-111.

19. Bocian A, Kopczynski J, Rieske P, et al. Simultaneous occurrence of medullary and papillary carcinomas of the thyroid gland with metastases of papillary carcinoma to the cervical lymph nodes and the coinciding small B-cell lymphocytic lymphoma of the lymph nodes-a case repor. Pol J Pathol. 2004; 55: 23-30.

20. Reid-Nicholson M, Moreira A, Ramalingam P. Cytologic features of mixed papillary carcinoma and chronic lymphocytic leukemia/small lymphocytic lymphoma of the thyroid gland. Diagn Cytopathol. 2008; 36: 813-817.

21. Shin J, Chute D, Milas M, et al. A rare case of chronic lymphocytic leukemia/small lymphocytic lymphoma presenting in the thyroid gland. Thyroid. 2010; 20: 1019-1023.

22. Trumper L, Matthaei-Maurer DU, Knauf W, et al. Centroblastic lymphoma of the thyroid supervening long-lasting chronic lymphocytic leukemia (B-CLL) demonstration of biclonality by immunohistochemical and gene rearrangement analysis. Klin Wochenschr 1988; 66: 736-742.

23. Reddy N, Thompson-Arildsen MA. Hodgkin's lymphoma: Richter's transformation of chronic lymphocytic leukemia involving the liver. J Clin Oncol. 2010; 28: 543-544.
24. Tadmor T, Bejar J, Attias D, et al. Richter syndrome in chornic lymphocytic leukaemia manifesting only as tumorous hepatomegaly. Britsh J Haematol. 2011; 155: 135.

25. Maryniak RK, Konecki R. 2 cases of Richter's syndrome. Acta Haematol Pol. 1991; 22: 165-169.

26. Reddy N, Thompson-Arildsen MA. Hodgkin's lymphoma: Richter's transformation of chronic lymphocytic leukemia involving the liver. J Clin Oncol. 2010; 28: 543-544.

27. A. Salihoglu, M. Ozbalak, D. Keskin, et al. An Unusual Presentation of a Chronic Lymphocytic Leukemia Patient with 17p Deletion After Reduced-Intensity Transplantation: Richter Syndrome and Concomitant Graft-Versus-Host Disease-Case Report. Transplantation Proceedings 2013; 45: 2845-2848.

28. Cohen Y, Da'as N, Libster D, et al. Large-cell transformation of chronic lymphocytic leukemia and follicular lymphoma during or soon after treatment with fludarabine-rituximab-containing regimens: natural history- or therapy-related complication? Eur J Haematol. 2002; 68: 80-83.

29. Janssens A, Berth M, de Paepe P, et al. EBV negative Richter's syndrome from a coexistent clone after salvage treatment with alemtuzumab in a CLL patient. Am J Hematol. 2006; 81: 706-712.

30. Dreger P, Dohner H, Ritgen M, et al. Allogeneic stem cell transplantation provides durable disease control in poor-risk chronic lymphocytic leukemia: long-term clinical and MRD results of the German CLL Study Group CLL3X trial. Blood 2010; 116: 2438-2447.

31. Kroft SH, Dawson DB, McKenna RW. Large cell lymphoma transformation of chronic lymphocytic leukemia/small lymphocytic lymphoma. A flow cytometric analysis of seven cases. Am J Clin Pathol. 2001; 115: 385-395.

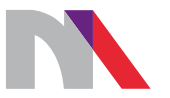

\section{Ministry of Science} and Higher Education

Republic of Poland

Generation of the DOI (Digital Object Identifier) - task financed under the agreement No. 618/P-DUN/2019 by the Minister of Science and Higher Education 